bmalinowska@tu.kielce.pl

\title{
SAVOIR VIVRE AN DEN HOCHSCHULEN - ÜBER DIE NOTWENDIGKEIT EINES NEUEN STUDIENFACHS IM ANGESICHT DER HERAUSFORDERUNGEN DES MODERNEN ARBEITSMARKTES
}

\begin{abstract}
Die aktuelle Arbeitsmarktentwicklung hat zur Folge, dass in die Studienprogramme an den Hochschulen neue Inhalte eingeführt werden müssen. Mit dem folgenden Beitrag wird beabsichtigt, das neue Fach Akademische gute Manieren $\mathrm{zu}$ präsentieren, die Gründe für dessen Einführung in den universitären Bereich und die Methoden für seine Umsetzung $\mathrm{zu}$ thematisieren. Das neue Fach wird im Kontext der Entwicklungen auf dem Arbeitsmarkt von heute und der komplizierten Situation der jungen Generation $Y$ dargestellt, deren Vertreter die notwendigen Soft Skills lernen müssen, um die beruflichen und gesellschaftlichen Herausforderungen der modernen Realität zu bewältigen.
\end{abstract}

Schlüsselwörter: Savoir vivre, Generation Y, Soft Skills, Arbeitsmarktentwicklung, moderne Gesellschaften

SAVOIR VIVRE IN HIGHER EDUCATION - ABOUT THE NECESSITY OF THE NEW ACADEMIC SUBJECT IN THE FACE OF THE CHALLENGES OF THE MODERN JOB MARKET

\section{ABSTRACT}

The modern job market development resulted in the necessity of introducing new programs in higher education. The paper undertakes the attempt at presenting the new subject Academic Good Manners, the reasons for its introduction in higher education programs and the methods 
of its realization. The new subject is being analyzed in the context of the today's job market development as well as the complicated situation of the young Generation $Y$, whose representatives need to learn the essential Soft Skills so as to be capable of embracing the professional and social challenges of the modern reality.

Keywords: savoir vivre, generation y, soft skills, job market development, modern societies

\section{EINFÜHRENDE BEMERKUNGEN}

An vielen polnischen Hochschulen werden in den letzten einigen Jahren „Gute Manieren“ als Studienfach angeboten. Nicht nur in Warszawa ${ }^{1}$ oder anderen Großstädten (vgl. Jagielnicka 2014) Polens, sondern auch in kleineren, um das Beispiel von Kielce ${ }^{2}$ zu nennen. Der Trend ist übrigens auch an den deutschen Hochschulen zu beobachten (vgl. Janert 2007) und entspricht der steigenden Nachfrage nach den sozialen Kompetenzen und persönlichen Fähigkeiten vor allem in der modernen Geschäftswelt (Bellon 2012). Die lange vernachlässigten Fragen der Höflichkeit und der guten Manieren sind heutzutage wieder stark im Kommen, weil sie neu interpretiert als eine der wichtigen Soft Skills verstanden und gebraucht werden.

Die Gründe für diese Entwicklung sind natürlich recht unterschiedlich. ${ }^{3}$ Nur einer davon wird jedoch als besonders relevant für die vorliegende Arbeit behandelt, nämlich die komplizierte Situation der Generation $Y$ auf dem Arbeitsmarkt von heute. Die aktuelle Arbeitsmarktentwicklung hat zur Folge, dass im akademischen Leben neue Inhalte in die Studienprogramme eingeführt werden müssen. Sowohl die Universitäten, als auch andere Hochschultypen, wie z.B. Technische Hochschulen, sehen es deswegen als eine ihrer wichtigsten neuen Aufgaben, ihren Studenten ein solches Studienprogramm anzubieten, das der unruhigen, sich ständig wandelnden, heutigen Realität gerecht wird, und den Stu-

1 Vgl. hierzu exemplarisch den Artikel von Chmielnicka E. Kodeks etyczny SGH Dobre obyczaje w nauczaniu, In: Gazeta SGH nr 183, https://www.akson.sgh.waw.pl, Zugang im 03.2016

2 Vgl. das Kommentar bei der Einführung des Studienfachs in „Gazeta Wyborcza” Naucza studentów dobrych manier. Pierwszy semestr: podstawy, szósty: oświadczyny., https://www.kielce. wyborcza.pl, Zugang im 03.2016.

3 Als Beispiel könnten in diesem Kontext die angestrebten Internationalisierungsprozesse an den Hochschulen genannt werden, vgl. Program Umiędzynarodowienia Szkolnictwa Wyższego, https:// www.nauka.gov.pl, Zugang im 03.2016. 
dierenden dazu verhilft, sowohl auf dem Arbeitsmarkt, als auch im Privatleben von heute und morgen sich problemlos zurechtzufinden.

Das Ziel des folgenden Beitrags ist, das neue Fach Akademische gute Manieren zu präsentieren, die Gründe für dessen Einführung in den universitären Bereich zu erörtern wie auch die Methoden für dessen Umsetzung näher zu bringen. Das neue Fach sollte dazu beitragen, dass sich die Studenten erstmal im akademischen Leben zurechtfinden, um später ihre Ziele auch im beruflichen Leben verwirklichen zu können. Das Konzept klingt so einfach wie es ist - Gute-Manieren-Kurse werden in das Studium integriert, um den Studenten, die im Moment als Vertreter der Generation $Y$ funktionieren, die heutzutage unentbehrlichen Soft Skills zu vermitteln, die in der Zeit der modernen Herausforderungen viel geschickter angewandt werden müssen als früher. Da die Entwicklungen und Erfahrungen in Polen und Deutschland in dieser Hinsicht einander ziemlich genau entsprechen, werden hiermit im Nachfolgenden die Meinungen sowohl der polnischen als auch der deutschen Experten und Publizisten zitiert. Als Paradebeispiel gilt das neue Studienprogramm an der Politechnika Świętokrzyska in Kielce, in das das Fach „Akademickie dobre wychowanie“ im Jahre 2013 eingeführt wurde und bis heute erfolgreich realisiert wird.

\section{GUTE-MANIEREN-KURSE - ZU DEN GRÜNDEN FÜR DIE EINFÜHRUNG DES NEUEN STUDIENFACHS}

Um genau zu verstehen, warum junge Erwachsene heute wieder Schulungen im Bereich gute Manieren brauchen, sollte man zuerst die Eigenschaften der Altersgruppe Generation $Y$ erfassen.

Die Generation $Y$ wird seit einigen Jahren intensiv erforscht und ganz unterschiedlich charakterisiert. Es handelt sich dabei im Allgemeinen um alle Menschen, die zwischen 1980-2000 geboren wurden und die gerade jetzt entweder studieren oder es versuchen, auf dem Arbeitsmarkt Fuß zu fassen. Es ist die erste Generation, für die der Umgang mit dem Internet zum Lebensalltag gehört. ${ }^{4}$ Charakteristisch für die Ypsiloner ist ihre starke und fast permanente Kontaktpflege mittels sozialer Netzwerke, sodass sie nicht selten Kontakte im echten Leben aus den Augen verlieren. Die Arbeitswelt nimmt die Generation als anspruchsvoll, flexibel und sehr selbstbewusst wahr (vgl. Kramer 2015). Es ist eine Generation, die vielleicht wohl als erste, sonderbare Ansprüche an die Arbeitgeber zu stellen versucht. Das schlägt sich u.a. darin nieder, dass sich die

4 Vgl. die Beschreibung der Generationen in dem Artikel: XYZ - Generationen auf dem Arbeitsmarkt, https://www.absolventa.de, Zugang: 03.2016. 
angehenden Arbeitnehmer beispielsweise nicht nur eine sinnvolle Arbeit und Abwechslung, sondern auch flexible Arbeitszeiten wünschen. Dabei sind sie nicht bereit, ihr ganzes Leben der beruflichen Sphäre zu unterordnen, denn Privatsphäre, Selbstverwirklichung und Unterhaltung sollen bei ihnen auf keinen Fall zu kurz kommen (ebd.). In der wohl schärfsten Kritikvariante bezeichnet man die Vertreter der Generation $Y$ als ,,verwöhnt, selbstverliebt, größenwahnsinnig und schlecht darin, sich selbst zu hinterfragen, dabei groß darin, sich zu überschätzen." (Bund 2014)

Die Ypsiloner selbst sehen sich folgendermaßen:

„Ich persönlich nehme meine Generation ganz anders wahr. Wir sind nicht faul. Wir wollen arbeiten. Nur anders. Mehr im Einklang mit unseren Bedürfnissen. Wir lassen uns im Job nicht versklaven, doch wenn wir von einer Sache überzeugt sind (und der Kaffeeautomat nicht streikt), geben wir alles. Wir suchen Sinn, Selbstverwirklichung und fordern Zeit für Familie und Freunde. Was wir verlangen, kommt einem gewaltigen Umbruch gleich: Wir fordern eine neue Berufswelt. In der alten herrscht vor allem Frust: Jeder vierte Beschäftigte in Deutschland hat innerlich gekündigt, 61 Prozent machen Dienst nach Vorschrift, wie aus dem 'Engagement Index ' des Beratungsunternehmens Gallup hervorgeht. In vielen Unternehmen gelten noch immer starre Arbeitszeiten und Präsenzpflichten. Statt Vertrauensarbeitszeit herrscht das Diktat der Stempeluhr. Feedback gibt es, wenn überhaupt, nur einmal im Jahr - beim obligatorischen Mitarbeitergespräch. Leistung wird daran bemessen, wie viele Stunden man bei der Arbeit verbringt, und nicht daran, was am Ende dabei herauskommt. In dieser Logik ist es sinnvoller, viel Zeit mit wenig Arbeit zu verbringen, anstatt viel Arbeit in kurzer Zeit zu erledigen. Langsames Arbeiten wird belohnt, schnelles Arbeiten bestraft." (ebd.)

Ein ähnliches Porträt, diesmal der polnischen Ypsiloner, schildert auch Sylwia Radzięta (2014). Ihr Fazit klingt dabei durchaus optimistisch, weil sie in der neuen Einstellung der jungen Arbeitnehmer vor allem die Chance auf neue positive berufliche Entwicklungen sieht (ebd.).

Bevor man also die Generation $Y$ voreilig als egoistisch und unrealistisch beurteilt, sollte noch ein wichtiger Aspekt der modernen Arbeitswelt in Erwägung gezogen werden und zwar der besonders schwierige Berufseinstieg der jungen Generation. Bernd Kramer schreibt zum Thema der Berufseinsteiger Folgendes:

„Der Berufseinstieg verläuft heute nicht einfacher, sondern schwieriger als früher. Befristete Arbeitsverträge unterschreiben überproportional häufig junge Arbeitnehmer. Unter den Unter-25-Jährigen haben 23 Prozent einen Zeitvertrag. Bei den Arbeitnehmern zwischen 25 und 35 Jahren sind es 13,8 Prozent. 
Das sind Zustände, die man sich in Westeuropa noch vor ein paar Jahrzehnten verbeten hätte. Inzwischen ragt Deutschland auch international heraus mit einer extrem hohen Befristungsquote bei jungen Berufstätigen: Nach Berechnung der Industrieländer-Organisation OECD werden junge Menschen lediglich in den Niederlanden noch häufiger mit einem Zeitvertrag abgespeist als in Deutschland. Selbst das Institut für Arbeitsmarkt- und Berufsforschung (IAB) spricht inzwischen von einer 'Umverteilung von Beschäftigungsrisiken hin zu jüngeren Beschäftigten'(Kramer 2015). Die Generation Y bezeichnet er so als eine Generation Prekär und unterstreicht den vernichtenden Einfluss „,der Erfahrung der Instabilität als Dauerzustand“ (ebd.), die für die Ypsiloner zum Alltag geworden ist.

Außer der wirtschaftlichen Komponente ist schließlich auch die menschliche Komponente zu berücksichtigen. Es handelt sich dabei um die Diskrepanz zwischen den potenziellen Vorgesetzten und Arbeitgebern, die oft früheren Generationen angehören - der jüngeren Generation X (Geburtsdatum zwischen 1960-1980) - und der älteren Generation der Baby Boomers (geboren zwischen 1943-1960) $)^{5}$, die völlig unterschiedliche Werte, Ziele und ein anderes Lebensgefühl vertreten. Der reibungslose Umgang miteinander ist trotz aller Unterschiede dennoch möglich, vorausgesetzt die Kommunikationsprozesse verlaufen mit genug Rücksichtnahme. Die Tatsache führt so zum nächsten Punkt des Beitrags der Bedeutung der Soft Skills.

Der Begriff Soft Skills ${ }^{6}$ bezieht sich auf solche Eigenschaften und Fähigkeiten, die außer dem fachlichen Wissen unentbehrlich sind, um Erfolge im beruflichen sowie privaten Leben erzielen zu können. Drei Sorten der Fähigkeiten werden im Allgemeinen unterschieden ${ }^{7}$ : persönliche, soziale und methodische Kompetenzen.

Unter persönlichen Kompetenzen versteht man beispielsweise Selbstbeobachtung und Selbstdisziplin (ebd.). Diese Fähigkeiten beziehen sich zwar nur indirekt auf die eigene Persönlichkeit, nicht desto weniger beeinflussen sie auch das ganze soziale Umfeld. Soziale Kompetenzen (ebd.), wie z.B. gute Menschenkenntnisse, Teamfähigkeit sowie ausgeprägtes Einfühlungsvermögen haben dagegen ganz direkte Auswirkungen auf andere Menschen.

5 Mehr dazu vgl. XYZ - Generationen auf dem Arbeitsmarkt, https://www.absolventa.de, Zugang: 03.2016 .

6 Vgl. hierzu die Definitionen: Soft Skills: Definition und Stellenwert, https://www.staufenbiel.ch, Soft Skills: Was steckt dahinter?, https://www.infoquelle.de, Zugang: 03.2016.

7 Vgl. hierzu genauere Beschreibungen der Fähigkeiten in dem Beitrag: Definition Soft Skills/Hard Skills Außerfachliche Kompetenzen zählen, https://www.berufsstrategie.de, Zugang: 03.2016. 
Von den methodischen Kompetenzen spricht man dann, wenn es sich einerseits um den geschickten Umgang mit neuen Medien handelt, andererseits assoziiert man damit solche Begriffe, wie Selbstmotivation und Selbstdisziplin sowie auch andere Fertigkeiten, die einem dazu verhelfen, problematische Sachverhalte zu lösen (ebd.).

Die Idee selbst, auch wenn der Name Soft Skills ganz modern klingt, ist nicht neu. Sie stammt von keinem geringeren als Adolph Freiherr Knigge, der in seinem 1788 veröffentlichten Werk „Über den Umgang mit Menschen“ einen Wunsch und Vorschlag geäußert hat, ein solches Leben zu führen, das „ein konfliktfreies, zivilisiertes und sinnerfülltes Miteinander ohne unnötige Reibungspunkte" ${ }^{\text {"8 }}$ garantiert. Wie man es heute immer noch in seinem Buch nachschlagen kann:

„Knigge ging es um ein ganzheitlicheres Bild verschiedenster Menschentypen, die miteinander Umgang hatten. Gerade wenn untere Schichten mit dem Adel in Berührung kamen, mussten sie wissen, was sie am Hofe erwartete. Im Grunde genommen war das Buch eher eine Weitergabe seines persönlichen Erfahrungsschatzes als ein Benimmwerk. Und dazu gehörte der Umgang mit einem gesunden Selbstbewusstsein und Wahrung der eigenen Würde ebenso wie auch Toleranz und Respekt gegenüber seinen Mitmenschen. Inhalte, die bis heute nichts an Aktualität eingebüßt haben. Egal, welchen Hierarchien man gegenübertreten muss." (ebd.)

Wie wichtig gute Umgangsformen im Geschäftsleben sind, kann man zahlreichen Webseiten entnehmen, die sich intensiv mit dem Thema auseinandersetzen..$^{9}$ Die bestätigt auch die zahlreiche populärwissenschaftliche Literatur, darunter viele Ratgeber. ${ }^{10}$ Werte wie: Höflichkeit, Zuvorkommenheit, Respekt, Toleranz und gegenseitige Rücksichtnahme sind so vom modernen Berufsleben kaum wegzudenken. Insofern ist das neue Angebot der Hochschulen für die Studierenden von großer Wichtigkeit.

8 Vgl. die Schlussfolgerungen in dem Artikel: Adolph Freiherr Knigge hats vorgemacht, https:// www.focus.de, Zugang: 03.2016.

9 Vgl. hierzu exemplarisch folgende Beiträge: Business Knigge Kompetenz ausstrahlen, https:// www.berufsstrategie.de und Warkentin N., Business-Knigge: Regeln im Beruf, https://www.karrierebibel.de, Zugang: 03.2016.

10 Vgl. hierzu exemplarisch: Sawicka, E.: Savoir-vivre Podręcznik dobrych manier, Warszawa -Bielsko-Biała 2011, Kamińska-Radomska, I.: Kultura biznesu Normy i formy, Warszawa 2011, Schneider-Flaig, S.: Der neue große Knigge Gutes Benehmen und richtige Umgangsformen, München 2013, Quittschau, A., Tabetnig, C.: Business-Knigge Die 100 wichtigsten Benimmregeln, Freiburg 2012 u.a. 


\section{CURRICULUM DES STUDIENFACHS ,AKADEMISCHE GUTE MANIEREN" AM BEISPIEL VON POLITECHNIKA ŚWIĘTOKRZYSKA IN KIELCE}

Der neue Kurs „Akademische gute Manieren“ wurde im Studienjahr 2013/2014 an der Technischen Universität in Kielce als Pflichtfach für alle Fachrichtungen eingeführt und wird in zwei Stufen unterrichtet. Die Studenten des ersten Semesters des Direktstudiums nehmen an den ersten fünf Stunden des Vortrags teil, die Fernstudenten haben im ersten Studienjahr drei Stunden Vortrag. Im sechsten Semester werden im Direktstudium weitere fünfzehn Stunden Unterricht angeboten, im Fernstudium gibt es dementsprechend zehn Stunden Vortrag.

Im ersten Teil des Kurses geht es zuallererst um die Bedeutung des Begriffs gute Manieren / gutes Benehmen. Den Studenten wird eine besondere Lebenseinstellung veranschaulicht, deren Kern ein in jeder Hinsicht respektvoller Umgang mit anderen Menschen ist. Den Rahmen für alle Höflichkeitsregeln und Rituale, die in darauffolgenden Vorträgen unterrichtet werden, schafft in diesem Kontext also der Respekt gegenüber anderen Menschen, deren mannigfaltigen Wertvorstellungen und Kulturen. Des Weiteren wird den Studenten der Einfluss des ersten Eindrucks erklärt, dessen Wichtigkeit die Studenten dazu ermuntern sollte, selbstständig und regelmäßig am eigenen Auftreten zu arbeiten. Außerdem werden richtige Verhaltensweisen bei der Begrüßung geübt. Von großer Bedeutung in der polnischen Gesellschaft ist z.B. die Antwort auf die Frage, wer wen zuerst grüßen sollte, beides in der Geschäftswelt und im Privatleben, aber auch von welcher Person in unterschiedlichen Situationen der Händedruck ausgeht. Weiterhin werden Strategien besprochen, die eine gelungene, positive Selbstdarstellung garantieren, sowie aktuell geltende Höflichkeitsregeln, die es einem ermöglichen, andere Menschen miteinander adäquat bekannt $\mathrm{zu}$ machen. Abgeschlossen wird die erste Vorlesungsreihe mit den bewussten und unbewussten Signalen der Körpersprache. Die positive Ausstrahlung eines Menschen, ist nämlich ein wichtiger Bestandteil eines bewusst aufgebauten Images.

Der Kurs wird im sechsten Semester (im Fernstudium gibt es den Kurs im siebten Semester) fortgesetzt. Die erste Vorlesung ist eine gute Gelegenheit dazu, das Basiswissen vom ersten Semester kurz auszuwerten. Zum zentralen Punkt des ersten Vortrags wird weiterhin die immer größere Rolle der interkulturellen Kompetenz. Das bedeutet eine umfangreiche Auseinandersetzung mit dem Begriff Kultur. Im kontrastiven Vergleich mit unterschiedlichen Sichtweisen 
und Werthaltungen anderer Gesellschaften werden die Studierenden mit der eigenen kulturellen Prägung konfrontiert, und dies mit dem Ziel auf die potentiellen Probleme und Konflikte hinzuweisen, die in der interkulturellen Kommunikation und Zusammenarbeit vorkommen könnten, wenn eine sensible und verständnisvolle Einstellung gegenüber anderen fehlt. Der Vortrag schafft so die Grundlage für die darauffolgenden Inhalte, die im Laufe des Semesters besprochen werden.

In den Vorträgen werden des Weiteren Themen besprochen, wie: die Benimmregeln bei Geschäftsessen oder privaten Einladungen, die Aufgaben des Gastgebers und die richtigen Verhaltensweisen als Gast, das vornehme Benehmen in einem schicken Restaurant, sowie der richtige Umgang mit den Gläsern und Besteck, darunter wird sowohl die Bestecksprache behandelt als auch alternative Besteckvarianten anderer Esskulturen, z.B. Stäbchen und Finger in der Rolle des Bestecks. Des Weiteren wird dem Business-Dresscode die Aufmerksamkeit geschenkt, mit der Berücksichtigung der Unterschiede zwischen Begriffen wie Trend und Stil. Es werden genaue Anweisungen gegeben, aus welchen Elementen ein Business-Outfit zusammengesetzt werden sollte, sowohl bei Frauen als auch bei Männern. Zu wichtigen Themenbereichen des Kurses gehören ferner die Umgangsformen, die bei Bewerbung zu beachten sind. Dabei werden nicht nur die Regeln besprochen, die im Bereich der Körpersprache vorkommen, z.B. eine souveräne und respektvolle Körperhaltung sowie eine freundliche Mimik, vielmehr werden die Teilnehmer auf die erhebliche Rolle der Kommunikation hingewiesen. Gemeint wird in diesem Kontext beispielsweise die richtige Anrede oder der geschickte Umgang der Kandidaten mit überraschenden und schwierigen Fragen, die bei Vorstellungsgesprächen keine Ausnahme sind. Die Höflichkeitsregeln am Arbeitsplatz werden zum Thema des vorletzten Vortrags. Pünktlichkeit, der respektvolle Umgang mit Kollegen, die Kunst des Small Talks, der Umgang mit geschäftlichen Telefonaten, sowie vertraulichen Informationen, das klare Trennen der Privatsphäre, aber auch die Ordnung am Arbeitsplatz sind die wesentlichen Punkte der Vorlesung. Die Vortragsreihe endet mit den zeitgemäßen Regeln der traditionellen, stilvollen Korrespondenz sowie der Netiquette. Das moderne Kommunizieren per E-Mail hat nämlich zum Teil ganz andere Verhaltensweisen in dem schriftlichen Briefwechsel hervorgebracht, die manchmal zu Missverständnissen führen können, besonders dann, wenn man Angewohnheiten aus dem Privatleben ins Geschäftliche importiert. 


\section{DIDAKTISCHE IMPLIKATIONEN AUS DER UNTERRICHTSPRAXIS DES FACHS „AKADEMISCHE GUTE MANIEREN"“}

Das im vorigen Kapitel dargestellte Rahmenprogramm wurde mit Berücksichtigung sowohl des aktuellen Wissenstands in dem Bereich des guten Benehmens als auch der gegenwärtigen Bedürfnisse und Anforderungen des modernen Arbeitsmarkts entwickelt. Unter didaktischen Implikationen wird in diesem Kontext vor allem die inhaltliche Planung und Strukturierung des Unterrichtsstoffes verstanden, sowie seine theoretische Einbettung und Analyse. Bei der Vorbereitung des Vorlesungsstoffes werden gewöhnlich folgende Materialien benötigt:

1. Das sog. Basiswissen, das vor allem Definitionen und Klassifikationen, sowie geschichtliche Hintergründe einer Sitte oder Verhaltensweise umfasst.

2. Die detaillierte Charakteristik der einzelnen Themenbereiche (mit zutreffenden Beispielen).

3. Die aktuellen statistischen Angaben, wenn sie für das Thema relevant sind (das ist z.B. beim Thema Bewerbung immer der Fall).

4. Presse- und Internetquellen, die zeitgemäße Fälle zitieren lassen, um die eine oder andere Regel zu testen.

Bei den Vorbereitungen auf jede Vorlesung ist es ratsam, sowohl an die Haupt- als auch an die Nebenzwecke kluge Überlegungen anzustellen, um vor allem das Wichtigste zum Vorschein bringen zu lassen. Da müssen z.B. immer wieder Entscheidungen getroffen werden, was in einem bestimmten Themenkreis auf jeden Fall von Bedeutung ist und worauf man aus Zeitgründen zu verzichten hat.

Um den Stoff möglichst günstig zu strukturieren, ist es nötig, jedes Mal einen Vortragsentwurf zu erstellen. Dies wird am folgenden Beispiel gezeigt:

Thema: Savoir vivre an der Universität, im Berufs- und Privatleben Einführende Bemerkungen
A. Definition des Begriffs savoir vivre
1. Respekt im Umgang mit anderen Menschen
2. Entscheidender Einfluss des ersten Eindrucks
B. Benimmregeln an Hochschulen 
1. Richtige Anrede bei Titeln

1. Angemessenes Begrüßen

2. Passendes Verhalten bei Vorlesungen und an anderen Orten des Universitätsgebäudes im Alltag

3. Geltende Höflichkeitsregeln bei der Kommunikation per E-Mail an der Hochschule

\section{DIE METHODIK DES NEUEN FACHS}

Unter Methodik versteht man hier die Organisation des Lehrens und des Lernens, die solche Aspekte wie Unterrichtsformat, dessen Verlaufsform, häufig angewandte methodische Grundformen, potentielle Rede- und Handlungssituationen sowie Sozialformen umfassen.

Das an der Technischen Universität Kielce für das neue Fach eingesetzte Lehrformat ist die Vorlesung. Das Format bedeutet Frontalunterricht, mit allen damit verbundenen Vor- und Nachteilen. Auf der Internetseite der Technischen Universität München findet man folgende Beschreibung der wesentlichen Merkmale des Formats:

„Zu den Stärken einer Vorlesung gehört, dass Sie einerseits eine große Anzahl von Studierenden gleichzeitig erreichen können, andererseits können Sie in die Systematik eines Fachgebietes einführen und einen Überblick über die relevanten Themen geben. Darüber hinaus wirkt die Vorlesung oft als rhythmisierendes und disziplinierendes Element im studentischen Alltag und stärkt als Begegnungsplattform das soziale Gruppengefüge unter den Studierenden. $\mathrm{Zu}$ den Schwächen dieses Formats gehört, dass es eine unverbindliche Konsumhaltung der Zuhörer fördert. Dies beruht insbesondere darauf, dass Studierende während der Vorlesung nur schwer persönlich einzubeziehen und zu aktivieren sind. Die Größe des Auditoriums und Faktoren wie unterschiedliches Vorwissen und Heterogenität erschweren es zusätzlich, die Situation positiv zu wenden. "11

Die älteste akademische Lehrmethode ist tatsächlich gut dazu geeignet, den Studierenden einen Überblick über das Thema: gute Manieren zu geben und sie in das Themengebiet einzuführen. Bei der Stoffvermittlung ist es durchaus möglich das Publikum mit den neuesten Erkenntnissen der Disziplin sowie dem aktuellen Diskussionsstand bekannt zu machen. Die Methode schafft auch Platz

11 Vgl. die Beschreibung des Begriffs im folgenden Beitrag: Vorlesung Das Flaggschiff der Hochschullehre, https://www.lehren.tum.de, Zugang:03.2016. 
für Experimentieren, wenn man zusammen mit den Studenten verschiedene Problemlösungen diskutiert. Man kann die Studierenden auch durch persönliche Ausstrahlung für das Thema begeistern und motivieren. Der geringe Kontakt zwischen dem Lehrenden und dem Lernenden ist jedoch beim Thema Manieren ein entscheidender Nachteil. Auch die Angewohnheit der Studenten, den Stoff einer Vorlesung automatisch passiv aufzunehmen, lässt sich nur schwer brechen, und erfordert viel Engagement von dem Lehrenden.

Bei der Vorbereitung der Vorlesungen werden in diesem Fall vor allem zwei Methoden bevorzugt: das deduktive und das problemhafte Vorgehen (vgl. Schumacher 2003). Das deduktive Vorgehen hat den Vorteil einer erheblichen Zeitersparnis, wenn zuerst die Theorie und die Höflichkeitsformen präsentiert werden, die dann mit Beispielen und besonderen Situationen unterstützt werden. Erfrischend wirkt die vereinzelt angewandte, routinebrechende, problemhafte Vorgehensweise. Bei der Präsentation der Themen werden die Studenten nämlich immer wieder darauf hingewiesen, dass sich die modernen Gesellschaften sehr schnell entwickeln, was einerseits das Entstehen neuer Regeln fördert, andererseits einige ältere Höflichkeitsrituale in Vergessenheit geraten lässt. Die allgemeine Schlussfolgerung, dass Manieren eine lebendige Erscheinung sind, und dass man die subtilen Unterschiede rechtzeitig erkennen und sich stets an die neue Realität anpassen muss, sowie die Tatsache, dass man selbst aktiver Teil dieser Prozesse ist, wirkt auf diese Weise motivierend und hilft die Neugierde und die Aufmerksamkeit der Studenten anzuregen und aufrechtzuerhalten.

Von großer Wichtigkeit bei der Arbeit mit der besonderen Generation $Y$ ist auch die Veranschaulichung der Sachverhalte durch visuelle und audiovisuelle Medien. Multimediale Präsentationen und Filme werden so zum wesentlichen Bestandteil einer Vorlesung.

Sehr viel Wert wird bei der Vorbereitung der Vorträge auch auf die Sprache gelegt. Nicht nur der Inhalt, sondern auch dessen lebendige Schilderung durch bildhafte Ausdrucksweise und richtige, nicht zu schwere, Wortschatzwahl können dazu beitragen, dass sich das Publikum über längere Zeit in das Thema engagiert. Überleitungssignale wie: „Nun komme ich zum zweiten Punkt ...“, „Besonders wichtig ist...“, „Abschließend möchte ich festhalten, dass..." können auf die einfachste Weise die fortlaufend sinkende Aufmerksamkeit der Zuhörer erhöhen. Sollten die Inhalte von den Studenten gemerkt werden, ist die Einsetzung der Redundanz notwendig. Bei den Wiederholungen der wichtigen Punkte und beim Paraphrasieren sollte man allerdings geschickt vorgehen, damit die Geduld des jungen Publikums nicht überstrapaziert wird.

Nicht außer Acht gelassen sollte die wohl effektivste Methode, die Aufmerksamkeit der Studenten aufrechtzuerhalten, und zwar das Ermuntern des 
Publikums zu einer bestimmten Frage Stellung zu nehmen und über verschiedene Meinungen zu diskutieren.

Eine der wichtigsten Strategien, unbeliebte oder sogar problematische Sachverhalte in den Vorlesungen anzugehen, ist schließlich der bewusste Einsatz von Humor. Damit werden vorsätzliche Humorstrategien gemeint, die einerseits das Wir-Gefühl im Publikum entwickeln können, andererseits eine Möglichkeit bieten, schwierige Situationen und Frustgefühle zu entschärfen und sich mit Tabu-Themen auseinanderzusetzen. Selbst ungünstige Mitteilungen und Kritik, die manchmal unvermeidlich ist, lassen sich mit Humor immer besser vermitteln (vgl. Rozmus 2010).

\section{ZUSAMMENFASSUNG}

Zusammenfassend kann man feststellen, dass das neue Fach in Anbetracht der Entwicklungen auf dem Arbeitsmarkt von heute eine richtige Bereicherung der Studienprogramme darstellt. Die Vertreter der jungen Generation $Y$ brauchen zweifellos nicht nur das nötige Fachwissen, sondern auch die notwendigen Soft Skills um die beruflichen und gesellschaftlichen Herausforderungen der modernen Realität zu bewältigen. Die praktische Vorbereitung der Vorlesungen scheint für die Lehrperson manchmal recht anspruchsvoll zu sein, weil die Inhalte von den Studierenden oft als persönlich eingestuft werden oder problematisch sind (das Vorwissen ist leider kaum vorhanden). Trotzdem lohnt sich die Mühe, denn nur auf diese Weise kann man zwischen Generationen Brücken bauen und eine Einigung erzielen, die die Zukunft nur positiv beeinflussen kann.

\section{LITERATURLISTE:}

„Adolph Freiherr Knigge hats vorgemacht“, Pristupljeno 03. 2016. URL: $<$ https://www.focus.de $>$.

Bellon, M. (2012). „Przez dobre maniery do sukcesu w biznesie”, Pristupljeno 03. 2016. URL: <https://www.kariera.pb.pl>.

Bund, K. (2014). „Wir sind jung...“, Pristupljeno 03. 2016. URL: $<$ https://www. zeit.de>.

„Business Knigge Kompetenz ausstrahlen“, Pristupljeno 03. 2016. URL: $<$ https://www.berufsstrategie.de $>$. 
Chmielnicka, E. „Kodeks etyczny SGH Dobre obyczaje w nauczaniu”, In: Gazeta SGH nr 183, Pristupljeno 03. 2016. URL: <https://www.akson.sgh. waw.pl>.

„Definition Soft Skills/ Hard Skills Außerfachliche Kompetenzen zählen“, Pristupljeno 03. 2016. URL: <https://www.berufsstrategie.de>.

Jagielnicka, U. (2014). „Studenci będą się uczyć dobrych manier”, Pristupljeno 03. 2016. URL: <https://www.wroclaw.pl>.

Janert, J. (2007). „Manieren studieren“, Pristupljeno 03. 2016. URL: <https:// www.faz.net>.

Kamińska-Radomska, I. (2011). Kultura biznesu Normy i formy, Warszawa.

Kramer, B. (2015). „Die Legende von der anspruchsvollen Generation Y“, Pristupljeno 03. 2016. URL: <https://www.spiegel.de>.

„Nauczą studentów dobrych manier. Pierwszy semestr: podstawy, szósty: oświadczyny." (2013). Pristupljeno 03. 2016. URL: <https://www.kielce. wyborcza.pl>.

„Program Umiędzynarodowienia Szkolnictwa Wyższego" (2015). Pristupljeno 03. 2016. URL: <https://www.nauka.gov.pl>.

Quittschau, A. Tabetnig, C. (2012). Business-Knigge Die 100 wichtigsten Benimmregeln, Freiburg.

Radzięta, S. (2014). „Czego spodziewać się po pokoleniu Y?” Pristupljeno 03. 2016. URL: <https://www.rynekpracy.pl>.

Rozmus, A. (2010). Wyktadowca doskonaty Podręcznik nauczyciela akademickiego, Warszawa.

Sawicka, E. (2011). Savoir-vivre Podręcznik dobrych manier, Warszawa Bielsko-Biała.

Schneider-Flaig, S. (2013). Der neue große Knigge. Gutes Benehmen und richtige Umgangsformen, München.

Schumacher, E. (2003). „Die Vorlesung Ideen und Konzepte für das Lernen und Lehren an Hochschulen“, Pristupljeno 03. 2016. URL: <https://www.hds. uni-leipzig.de>.

Schmidt J. (2015). „Soft Skills: Definition und Stellenwert“, Pristupljeno 03. 2016. URL: <https://www.staufenbiel.ch>.

„Soft Skills: Was steckt dahinter?“, Pristupljeno 03. 2016. URL: $<$ https://www. infoquelle.de>.

„Vorlesung Das Flaggschiff der Hochschullehre“, Pristupljeno 03. 2016. URL: $<$ https://www.lehren.tum.de $>$.

Warkentin, N. (2016). „Business-Knigge: Regeln im Beruf“, Pristupljeno 03. 2016. URL: <https://www.karrierebibel.de>. 
„XYZ - Generationen auf dem Arbeitsmarkt“, Pristupljeno 03. 2016. URL: $<$ https://www.absolventa.de $>$.

\title{
Barbara Maj-Malinowska
}

\section{SAVOIR VIVRE IN HIGHER EDUCATION - ABOUT THE NECESSITY OF THE NEW ACADEMIC SUBJECT IN THE FACE OF THE CHALLENGES OF THE MODERN JOB MARKET}

\begin{abstract}
Summary
The modern job market development resulted in the necessity of introducing new programs in higher education. The paper undertakes the attempt at presenting the new subject Academic Good Manners, the reasons for its introduction in higher education programs and the methods of its realization. The new subject is being analysed in the context of the today's job market development as well as the complicated situation of the young Generation Y, whose representatives need to learn the essential Soft Skills so as to be capable of embracing the professional and social challenges of the modern reality.
\end{abstract}

Keywords: savoir vivre, generation y, soft skills, job market development, modern societies

Received: 28 April 2016, Accepted: 14 July 2016 